\title{
Radiofrequency Ablation in Treatment of Atrial Fibrillation
}

\author{
R. LÁBROVÁ ${ }^{1}$, J. ŠPINAR ${ }^{1}$, N. HONZÍKOVÁ ${ }^{2}$ \\ ${ }^{1}$ Department of Internal Medicine - Cardiology, Faculty Hospital Brno and Faculty of Medicine, \\ Masaryk University, Brno, Czech Republic, ${ }^{2}$ Department of Physiology, Faculty of Medicine, \\ Masaryk University, Brno, Czech Republic
}

Received February 8, 2010

Accepted March 26, 2010

\begin{abstract}
Summary
Beside heart failure and metabolic syndrome, atrial fibrillation is termed the cardiovascular epidemic of the $21^{\text {st }}$ century. Its increased morbidity and mortality is alarming. The present, most effective therapy of atrial fibrillation is catheter ablation. Successful ablation of atrial fibrillation prevents the occurrence and progression of electrical, structural and mechanic myocardium remodelling, improves function of the left ventricle, and prevents the risk of thrombembolism. Onset of sinus rhythm activates the reversal remodelling leading to wall reconstruction and atrium reduction. The paper reviews the technique and presents own experience with catheter ablation.
\end{abstract}

\section{Key words}

Atrial fibrillation • Catheter ablation

\section{Corresponding author}

R. Labrova, Department of Internal Medicine - Cardiology, Faculty Hospital Brno and Faculty of Medicine, Masaryk University, Jihlavská 20, 62500 Brno, Czech Republic. Phone: +420-532 232 160, Fax: +420-532 232 611. E-mail: labrova@seznam.cz

\section{Introduction}

Atrial fibrillation (AF) is the most frequent arrhythmia. Its prevalence in the adult population reaches $1.0 \%$ and rises with age (Go et al. 2001, Feinberg et al. 1995). However, this increase cannot be explained exclusively by ageing of the population. Rising AF incidence becomes a real health, social and economic problem. AF often occurs in consequence of heart failure and depends on its severity (Maisel and Stevenson 2003, Fuster et al. 2006). Successful AF therapy and a recovery of sinus rhythm (SR) improve and sometimes completely normalize the left ventricular function (Gentlesk et al. 2007). In younger patients paroxysmal forms prevail; in elderly, AF gradually changes due to electrical and structural remodelling of the left atrium into a persistent or even permanent form (Furberg et al. 1994).

Haïssaguerre et al. (1998) postulated in 1998 that one or more ectopic focuses exist, mostly in the atrial myocardium at the pulmonary vein orifices, exceptionally also at other localizations. Moreover, for AF to persist, the existence is essential of one or more re-entry circuits (the so called rotors), localized in the posterior wall left atrium as well as in pulmonary vein.

Recently, intensive research has been focused mainly on changes in genetic expression of the ion channels affecting the action potential of atrial cardiomyocytes and on molecular alterations related to AF. Possible genetic defects, underlying the familiar form of arrhythmia or a certain predisposition for its occurrence (Brugada 2004) have been studied as well. It is disputable if the molecular changes are the very reason of AF occurrence or its consequence only, i.e. if they result from adaptation or compensation mechanisms during AF.

Electrical atrial remodelling in atrial fibrillation occurs within minutes to days. It is caused by changes of ionic channels and thereby electrical currents namely of potassium and calcium. Some of these channelopathies are genetically conditioned. In chronic AF, morphological changes underlying structural remodelling appear during a few months. Atrial dilatation and 
hypertrophy, myocyte hypertrophy, myolysis, fibrotic changes, and fat infiltration are the most frequent symptoms. Connective tissues deposits among atrial myocytes lead to marked electrophysiological nonhomogeneities, decelerating conductance in the atrium and thus initiate the development of the mother re-entry circuits (Feng et al. 1998, Douglas 2002). Certain changes probably arise from ageing, degenerative changes or from activation of the renin-angiotensinaldosterone system (RAAS). Some changes underlying structural remodelling are reversible, but their reversibility may be incomplete. Prevention of the structural changes increases probability of recovery and maintaining of sinus rhythm.

As a consequence of structural remodelling, mechanical left atrium function is reduced. Organized mechanical contraction is absent; output rate of the left atrium auricle as a measure of its thrombogeneity is reduced. Conditions for blood stasis and thrombus are set up and thus the risk of thrombembolism increases. Due to missing atrial contribution, the cardiac output may be reduced up to $20 \%$.

\section{Treatment of atrial fibrillation}

Epidemiologic estimates of AF growing incidence are alarming and therefore, it is necessary to propose adequate strategy prevention and treatment of AF. Pharmacologic therapy still remains the first choice treatment of AF. Efficiency of this antiarrhythmic therapy ranges from 40 to $60 \%$; it is in most cases of temporary character and is accompanied by a number of adverse effects. The risk of the most serious pro-arrhythmic effect of the antiarrhythmics rises with severity of structural heart remodelling. The patients with heart remodelling may be administered either class I antiarrhythmics for rhythm control and/or in combination with beta blocker or calcium channel blocker, or class III antiarrhythmics combined with beta blocker. The patients with heart failure $(\mathrm{HF})$ and ejection fraction under $35 \%$ are treated either with class III antiarrhythmics only or in combination with beta blocker. Antiarrhythmics, decelerating AV conduction - calcium blockers, beta blockers and/or digoxine - are administered in patients with AF without present organic heart disorder.

Atrial remodelling, AF recurrence and persistence may be affected by pharmacological means, e.g. statins, corticoids and namely RAAS blocking agents (Calo et al. 2005, Dernellis and Panaretou 2004, Young-
Xu et al. 2003, Brown and Vaughan 2000).

If the abovementioned combined therapy of AF fails, the non-pharmacological procedures have to be applied. The most rapidly expending method has been selective catheter ablations of symptomatic, paroxysmal and even chronic AF forms accompanied by heart failure symptoms.

\section{The origins of ablation technique}

The first radiofrequency catheter ablation of AF patterned the surgical procedure labelled MAZE, a system of linear incisures and sutures carried out in both left and right atrium. This procedure used to be mazy indeed, and ensued by a number of complications. The efficiency of the more accessible the right atrium catheter ablation was empirically found less effective and finally abandoned (Haïssaguerre et al. 1994, 1996).

Since the discovery of focal origin of the AF, the catheter ablations concentrated at the sites of origin in central parts of pulmonary veins (Haïssaguerre et al. 2000, Lábrová and Špinar 2008). These focal ablations were soon deserted because of a great risk of severe pulmonary vein stenosis. Moreover, some of the ectopies were usually missed and caused a relapse of AF.

\section{Biophysical aspects of radio-frequency ablation}

Radio-frequency energy (RFE) applied through the percutaneous transvenous catheter has been used in the therapy of arrhythmias since 1987. This energy turned into heat, abolishes the abnormal conductance of the tissue and thus eliminates the arrhythmia. RFE is generated by a continuous non-modulated alternating $500 \mathrm{kHz}$ current. The mechanism of RFE generates the temperature between $40-55{ }^{\circ} \mathrm{C}$. The cells desiccate and undergo coagulation necrosis resulting in a small homogeneous lesion. Most of the thermic injury concerns an area only slightly exceeding the diameter of the ablation electrode. The size of the lesion depends on the temperature at the boundary between the electrode and the endocardium, on the diameter and length of the distal (tip) electrode and on the heat loss through the blood stream.

The temperature at the electrode/tissue interface increases during 6-15 sec of RFE application. At that time, equilibrium is reached among heat production, conduction and radiation. In the case of insufficient 
contact between the electrode and the tissue, the impedance increases and the input of RFE is automatically stopped.

\section{Introductory examinations}

One day prior to the intervention, the patient undergoes transoesophageal echocardiographic examination to assess the left atrial size and function. The patient is heparinised during the intervention and blood coagulation is controlled. Then, under local anaesthesia, 3D X-ray and ECG control, ten-polar exploratory catheter is introduced into the coronary sinus via right jugular vein or left femoral vein (Seldinger technique). The catheter examines and monitors the electrical activity. At the same time, it drives a programmable pacemaker. Next, two long sheaths are introduced into the left atrium via right femoral vein and transseptal puncture. The sheaths serve to insert the ablation and mapping (LASSO) catheters into the pulmonary vein orifice.

Immediately before ablation the pulmonary vein angiography is carried out in order to evaluate the anatomy of the pulmonary veins, and in particular of their orifices. Thereby the data for optimum location of the circular mapping catheter are obtained.

\section{Methods of catheter ablation}

The aim of the present catheter ablation of paroxysmal AF (PAF) is to eliminate all arrhythmogenic focuses in all four pulmonary veins and at the same time, to get clear of the maternal re-entry circuits (rotors) that might trigger and sustain PAF (Marrouche et al. 2002). In search for greater efficiency, some authors carry out linear ablations or focal lesions in order to affect re-entry circuits or autonomic ganglia in the left atrium (Oral et al. 2006). Nevertheless, the basis of the invasive treatment of the AF is ablation of pulmonary veins.

The conventionally used method of catheter ablation was developed in Bordeaux and is electrophysiological in principle. Its purpose is to achieve electrical isolation of the pulmonary veins (Fig. 1). Their electrical activity is monitored by means of the mapping circular LASSO catheter successively introduced into the vein orifices. The conventional ablation is directed around orifices under continuous control of the electrical signals from the pulmonary veins and under X-ray control. The electrical isolation is achieved by interruption of the conducting fibres which join the left atrium muscle and the pulmonary vein orifice. As soon as the isolation is achieved, the electrical activity recorded by LASSO catheter in the pulmonary vein discontinues. If the connecting fibres are fragmented and irregularly organized, ablation may be carried out segmentally, thus saving the radio-frequency energy (RFE). This method is successful in $70-80 \%$ of the cases; the risk of pulmonary vein stenosis is negligible, $0.5-2 \%$ (Tsang et al. 2003).

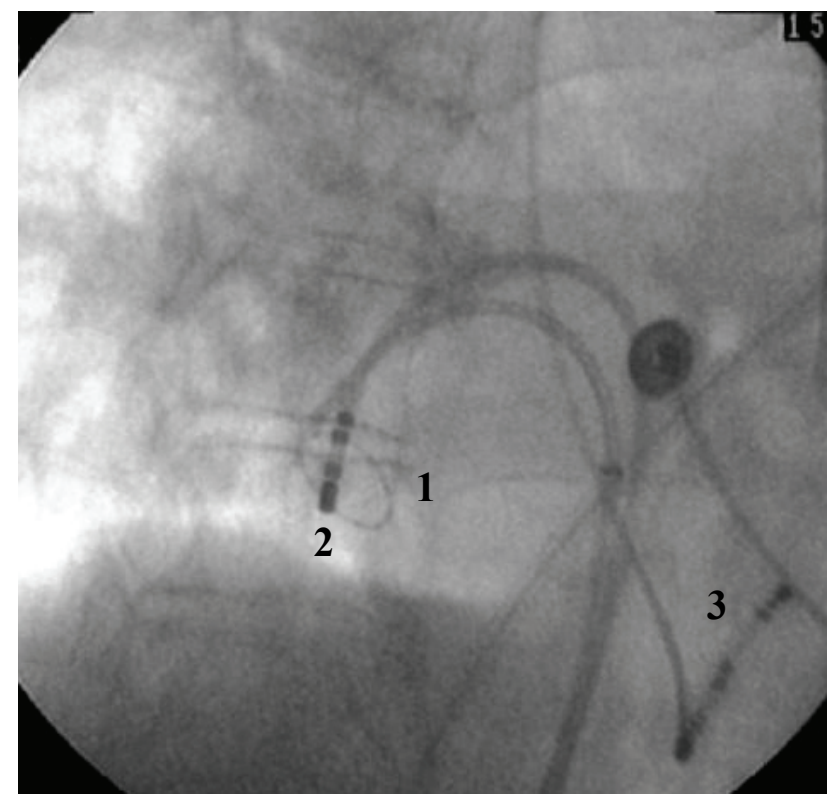

Fig. 1. Fluoroscopy right anterior oblique (RAO) views of the heart and position of catheters. 1-mapping circular Lasso catheter in the ostium of the right lower pulmonary vein; 2-quadripolar mapping/ablation catheter in the ostium of the right lower pulmonary vein; 3-decapolar mapping catheter in the coronary sinus.

Simultaneously with the Bordeaux method, another one was introduced in Milano. It is based on 3D electroanatomical mapping by means of specially designed system CARTO. RFE is applied in broader continuous lines around the venous orifices (Pappone et al. 2001). The circular lesions are produced at the dorsal wall of the left atrium thereby disconnecting the re-entry circuits around the orifices as well as disorganizing the rotors and even the vagal ganglia. This method is successful in $70-90 \%$ of the cases. The optimum resolution of PAF appears to be a combination of both methods as used also at our department. The achieved efficiency is between 80-90 \% (Ouyang et al. 2004).

The intervention is carried out during running fibrillation, sinus rhythm or atrial stimulation. The very ablation is performed by a $7 \mathrm{~F}$ catheter with cooled-tip electrode. The cooling is monitored and regulated at 


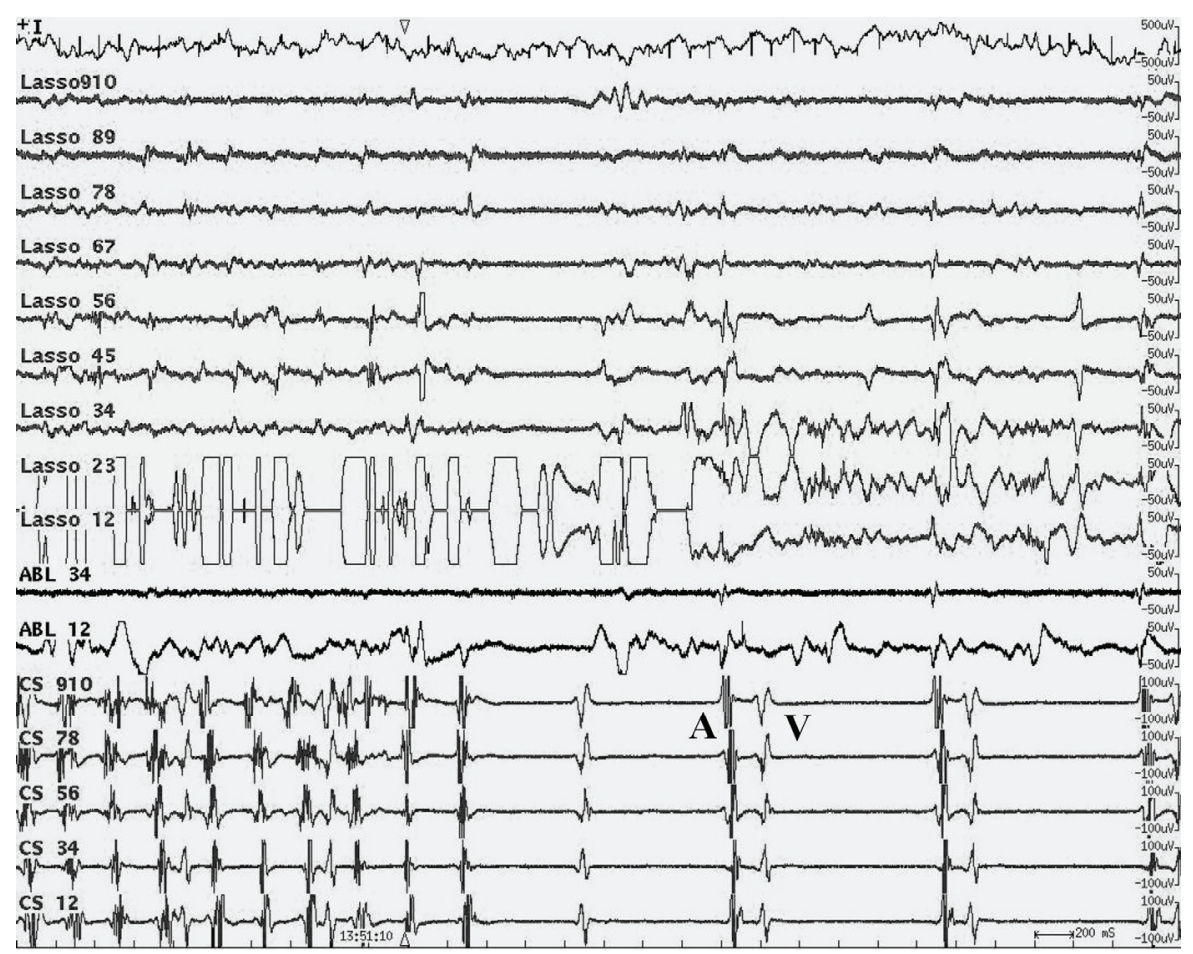

Fig. 2. Intracardiac ECG record. CS left atrial activity recorded from coronary sinus by exploratory catheter: initial paroxysmal fibrillation followed by sinus rhythm due to radiofrequency energy application (arrow). LASSO catheter scans fast irregular atrial activity in pulmonary vein throughout. A - left atrium, $V$ left ventricle, $A B L$ - records from ablation catheter. temperatures below $43{ }^{\circ} \mathrm{C}$. The power used ranges between $15-30 \mathrm{~W}$.

The application of RFE last as a rule $30-60 \mathrm{sec}$ and is several times repeated. The procedure is painless, only very seldom the mild chest distress is reported.

Segmental ablation is focused on the particular fascicle of muscle fibres connecting left atrium and the vein. The ectopic activity remains isolated at the pulmonary vein. The electrical activity monitored in the vein decreases within RFE application, and eventually disappears (Fig. 2). If RFA is carried out during AF, the sinus rhythm is regularly restored. Last, the effect of ablation is checked in all pulmonary vein using the mapping catheter. In the case of residual electrical activity, representing a risk of AF relapse, ablation is repeated.

In case of chronic AF forms the arrhythmogenic substrate is more complex, because of present atrial remodelling.

In the majority of cases a large lesion is produced around the pulmonary veins, completed by a linear lesions and ablation of fractionated potentials in the left atrium.

\section{Our results}

Between 2004 and 2008, 126 catheter ablations of symptomatic paroxysmal AF were carried out at our department and statistically evaluated. In the case of inefficiency of the pharmacological therapy the radiofrequency catheter ablation was indicated. Initially, the conventional method was used, i.e. the segmental catheter ablation without wide circular lesions. In 2006 the second ablation strategy was introduced, employing the electroanatomical mapping by the CARTO system (Fig. 3). Electric isolation of all four pulmonary veins was the chief objective of both methods (Fig. 4).

The set of 126 patients comprised 41 women (33\%) and 85 men (67\%), average age of $52.9 \pm 9.1$ years (31-76); follow up was carried out for $30.7 \pm 14.6$ months (6-57). In the most cases the patients did not suffer from any significant organic heart affection, except moderately dilated left atrium. Hypertension was the most frequent risk factor - nearly in three fourths of the patients (Table 1). Median of the number of RF energy applications was 39 minutes, radiation time length - 41.9 minutes, performance lengths - 213 minutes. Rate of success of the ablation in our set is comparable with the available literature sources. Rate of success assessed by the 24hour ECG Holter monitoring was as follows: in $2004-$ $87.5 \%$, in $2005-77.4 \%$, in $2006-79.2 \%$, in $2007-$ $80.1 \%$ and in $2008-83.2 \%$. Recurrence of AF is defined as its duration above 30 seconds. The number of patients with pharmacological antiarrhythmic therapy was reduced after successful catheter ablation (Table 2). The number of patients without any antiarrhythmic therapy increased significantly after the catheter ablation, from $3 \%$ before the ablation up to $25 \%$ after the intervention. 
The number of patients for double combination of the antiarrhythmic therapy was increased significantly - from $41 \%$ before to $15 \%$ after ablation. After the catheter ablation there is no difference in use of one drug, but less aggressive antiarrhythmics were applied. There was also no difference in patients taking three drugs, who underwent unsuccessful intervention and who were subject to repeated RFA. After the second intervention
3 patients were free from any antiarrhythmic therapy. After ablation use of the beta blockers remains unchanged, but the patients are administered statistically significantly reduced drug quantities - amiodarone (by $22 \%$ ), propaphenon (by $12 \%$ ), digitalis (by $14 \%$ ) and sotalol (by $17 \%$ ). The number of patients, with chronic anticoagulation therapy, was also significantly reduced after ablation.

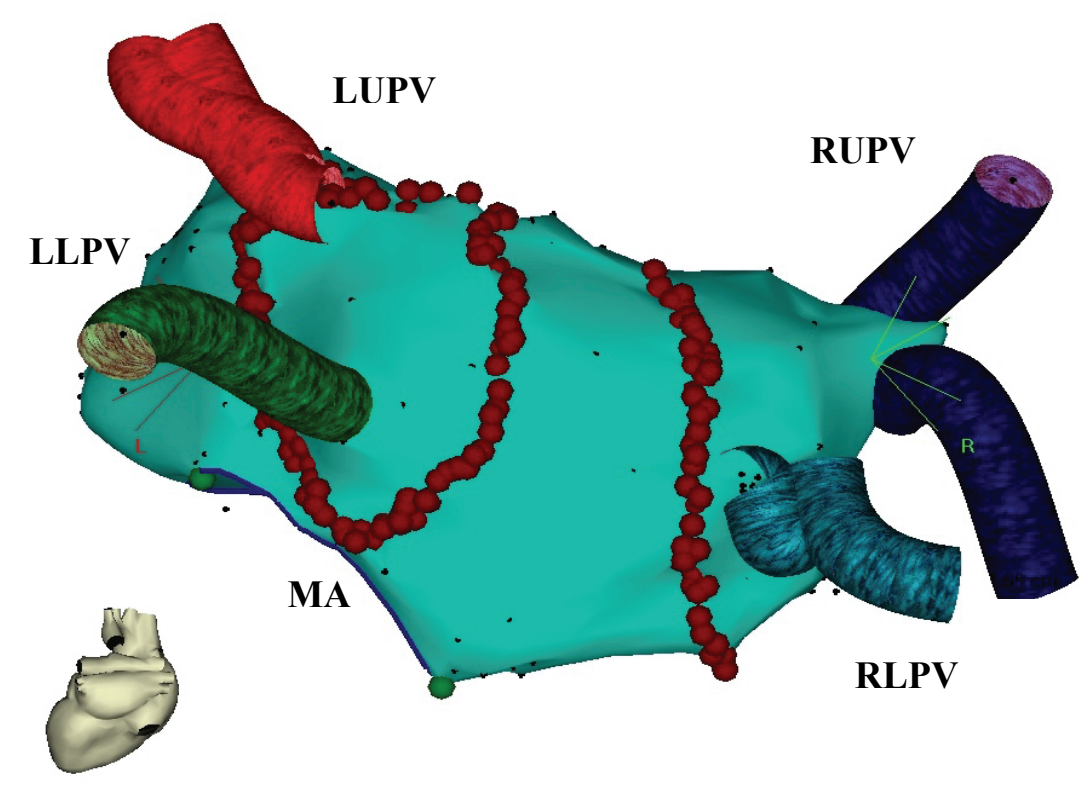

Fig. 3. Electroanatomic circumferential lesions. The pulmonary veins were continuously encircled by electroanatomically navigated RF lesions common for the ipsilateral veins. LUPV - left upper, LLPV - left lower, RUPV - right upper, RLPV - right lower pulmonary vein, MA - mitral annulus

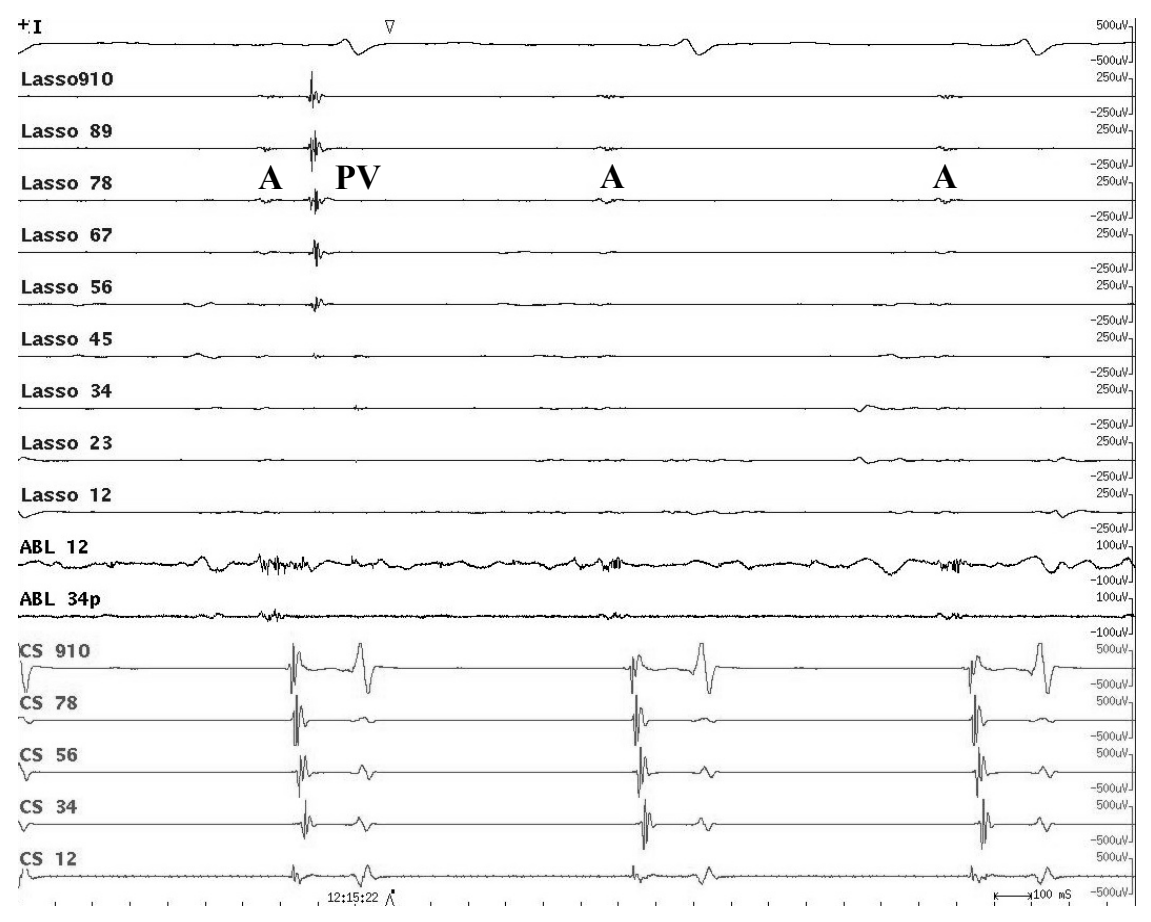

Fig. 4. Intracardiac ECG from left atrium (A) and pulmonary vein (PV) recorded by LASSO catheter. Activity of $A$ remains after electrical isolation of PV (arrow) while that from PV disappears. ABL - records from ablation catheter. 
Table 1. Clinical characteristics.

\begin{tabular}{lc}
\hline Patients (females) & $126(41)$ \\
Age (years) & $52 \pm 9(31-76)$ \\
History of AF (years) & $7.3 \pm 4.2(1-21)$ \\
Left ventricular ejection fraction (\%) & $60 \pm 5(40-75)$ \\
Left atrial transverse diameter (mm) & $43 \pm 6(35-48)$ \\
Arterial hypertension & $92(73 \%)$ \\
Hyperliporoteinemia & $66(52 \%)$ \\
Diastolic dysfunction & $33(26 \%)$ \\
Hyperthyroidism & $16(13 \%)$ \\
Hypothyroidism & $15(12 \%)$ \\
Diabetes mellitus & $16(13 \%)$ \\
Mitral regurgitation grade I-II & $13(10 \%)$ \\
Coronary heart disease & $13(10 \%)$ \\
Previous stroke & $6(5 \%)$ \\
Arrhythmia-induced cardiomyopathy & $6(5 \%)$ \\
\hline
\end{tabular}

In two women, severe complications occurred and were, settled without any permanent consequences. Cerebellar embolization and brain oedema was necessary to treat transient drainage in one patient and hemopericardium in another one within 48 hours after the intervention had to be resolved surgically.

\section{Conclusions}

The atrial fibrillation therapy has to be complex and individually adjusted with respect to accompanying illness, e.g. hypertension, heart failure or coagulopathy. Catheter ablation became nowadays the most effective therapy. Successful ablation of the atrial fibrillation
Table 2. Antiarrhythmic medication before and after ablation.

\begin{tabular}{llll}
\hline & $\begin{array}{l}\text { Before } \\
\text { ablation }\end{array}$ & $\begin{array}{l}\text { After } \\
\text { ablation }\end{array}$ & P-value \\
\hline No AAD & $4(3 \%)$ & $32(25 \%)$ & $<0.0001$ \\
1 AAD & $57(45 \%)$ & $64(51 \%)$ & $\mathrm{NS}$ \\
2 AADs & $52(41 \%)$ & $19(15 \%)$ & $<0.0001$ \\
3 AADs & $13(10 \%)$ & $11(9 \%)$ & $\mathrm{NS}$ \\
Beta blocker & $53(42 \%)$ & $49(39 \%)$ & $\mathrm{NS}$ \\
Verapamil & $4(3 \%)$ & $8(6 \%)$ & $\mathrm{NS}$ \\
Digoxin & $34(27 \%)$ & $17(13 \%)$ & $<0.05$ \\
Propafenone & $36(29 \%)$ & $21(17 \%)$ & $<0.05$ \\
Sotalol & $34(27 \%)$ & $13(10 \%)$ & $<0.01$ \\
Amiodarone & $49(39 \%)$ & $21(17 \%)$ & $<0.001$ \\
\hline
\end{tabular}

Abbreviations: $A A D=$ antiarrhythmic drug; number of patients and \%; significance of values after vs. before ablation

prevents occurrence and progression of electrical, structural and mechanic myocardium remodelling, improves function of the left ventricle. The onset of sinus rhythm initiates the reversal of remodelling. With increasing experience and upgrading technology, the RF catheter ablation of the atrial fibrillation becomes widely available and most effective therapy of symptomatic patients.

\section{Conflict of Interest}

There is no conflict of interest.

\section{Acknowledgements}

Supported by grant IGA MZ NR 9337-3.

\section{References}

BROWN NJ, VAUGHAN DE: Prothrombotic effect of angiotensin. Adv Intern med 45: 419-429, 2000.

BRUGADA R: Molecular biology of atrial fibrillation. Minerva Cardioangiol 52: 188-199, 2004.

CALO L, BIANCONI L, COLIVICCHI F, LAMBERTI F, LORICCHIO ML, DE RUVO E, MEO A, PANDOZI C, STAIBANO M, SANTINI M: N-3 fatty acids for the prevention of atrial fibrillation after coronary artery bypass surgery. J Am Coll Cardiol 45: 1723-1728, 2005.

DERNELLIS J, PANARETOU M. Relationship between C-reactive protein concentrations during glucocorticoid therapy and recurrent atrial fibrillation. Eur Heart J 25: 1100-1107, 2004.

DOUGLAS P: The left atrium: a biomarker of chronic diastolic dysfunction and cardiovascular disease risk. $J$ Am Coll Cardiol 42: 1206-1207, 2002.

FEINBERG WM, BLACKSHEAR JL, LAUPACIS A, KRONMAL R, HART RG: Prevalence, age distribution, and gender of patients with atrial fibrillation. Analysis and implications. Arch Intern Med 155: 469-473, 1995.

FENG J, YUE L, WANG Z, NATTEL S: Ionic mechanism of regional action potential heterogeneity in the canine right atrium. Circ Res 83: 541-551, 1998. 
FURBERG CD, PSATY BM, MANOLIO TA, GARDIN JM, SMITH VE, RAUTAHARJU PM: Prevalence of atrial fibrillation in elderly subject (The Copenhagen City Heart Study). Am J Cardiol 74: 236-241, 1994.

FUSTER V, RYDEN LE, CANNOM DS CRIJNS HJ, CURTIS AB, ELLENBOGEN KA: ACC/AHA/ESC 2006 quidelines for the management of patients with atrial fibrillation. Eur Heart J. 27: 1976-2030, 2006.

GENTLESK PJ, SAUER WH, GERSTENFELD EP, LIN D, DIXIT S, ZADA E, CALLANS. D, MARCHLINSKI FE: Reversal of left ventricular dysfunction following ablation of atrial fibrillation. $J$ Cardiovasc Electophysiol 18: 9-14, 2007.

GO AS, HYLEK EM, PHILLIPS KA, CHANG Y, HENAULT LE, SELBY JV, SINGER DE: Prevalence of diagnosed atrial fibrillation in adults. National implications for rhythm management and stroke prevention: the anticoagulation and risk factors in atrial fibrillation (ATRIA) Study. JAMA 285: 2370-2375, 2001.

HAÏSSAGUERRE M, GENCEL L, FISCHER B, LE MÉTAYER P. POQUET F, MARCUS FI, CLÉMENTY J: Successful catheter ablation of atrial fibrillation. J Cardiovasc Electrophysiol 5: 1045-1052, 1994.

HAÏSSAGUERRE M, JAÏS P, SHAH DC, GENCEL L, PRADEAU V, GARRIGUES S: Right and left atrial radiofrequency catheter therapy of paroxysmal atrial fibrillation. J Cardiovasc Electrophysiol 7: 1132-1144, 1996.

HAÏSSAGUERRE M, JAÏS P, SHAH DC, TAKAHASHI A, HOCINI M, QUINIOU G, GARRIGUE S: Spontaneous initiation of atrial fibrillation by ectopic beats originating in the pulmonary veins. $N$ Engl J Med 339: 659-666, 1998.

HAÏSSAGUERRE M, SHAH DC, JAÏS P, HOCINI M, YAMANE T, DEISENHOFER I: Electrophysiological breakthroughs from the left atrium to the pulmonary veins. Circulation 101: 1409-1417, 2000.

LÁBROVÁ R, ŠPINAR J: Catheter ablation of atrial fibrillation. Kardiol Rev 10: 103-110, 2008.

MAISEL WH, STEVENSON LW: Atrial fibrillation in heart failure: epidemiology, patopfysiology, and rationale for therapy. Am J Cardiol 20 (Suppl D): 2-8, 2003.

MARROUCHE NF, DRESING T, COLE C, BASH D, SAAD E, BALABAN K: Circular mapping and ablation of the pulmonary vein for treatment of atrial fibrillation. J Am Coll Cardiol 40: 464-474, 2002.

ORAL H, CHUGH A, GOOD E, SANKARAN S, REICH SS, IGIC P, ELMOUCHI D: A tailored approach to catheter ablation of paroxysmal atrial fibrillation. Circulation 113: 1824-1831, 2006.

OUYANG F, BÄNSCH D, ERNST S, SCHAUMANN A, HACHIYA H, CHEN ML: Complete isolation of the left atrium surrounding the pulmonary veins: new insights from the double-lasso technique in paroxysmal atrial fibrillation. Circulation 110: 2090-2096, 2004.

PAPPONE C, ORETO G, ROSANIO S, VICEDOMINI G, TOCCHI M, GUGLIOTTA F, SALVATI A, DICANDIA C: Atrial electroanatomic remodeling after circumferential radiofrequency pulmonary vein ablation. Efficacy of an anatomic approach in a large cohort of patients with atrial fibrillation. Circulation 104: 2539-2544, 2001.

TSANG TS, PETTY GW, BARNES ME, O'FALLON WM, BAILEY KR, WIEBERS DO, SICKS JD, CHRISTIANSON TJ, SEWARD JB, GERSH BJ.: The prevalence of atrial fibrillation in incident stroke cases and matched population controls in Rochester, Minnesota: changes over three decades. J Am Coll Cardiol 42: 93-100, 2003.

YOUNG-XU Y, JABBOUR S, GOLDBERG R, BLATT CM, GRABOYS T, BILCHIK B: Usefulness of statin drugs in protecting against atrial fibrillation in patients with coronary artery disease. Am J Cardiol 92: 1379-1383, 2003. 